\title{
TROPICAL MIXTURES OF STAR TREE METRICS
}

\author{
MARÍA ANGÉLICA CUETO
}

\begin{abstract}
We study tree metrics that can be realized as a mixture of two star tree metrics. We prove that the only trees admitting such a decomposition are the ones coming from a tree with at most one internal edge, and whose weight satisfies certain linear inequalities. We also characterize the fibers of the corresponding mixture map. In addition, we discuss the general framework of tropical secant varieties and we interpret our results within this setting. Finally, we show that the set of tree metric ranks of metrics on $n$ taxa is unbounded.
\end{abstract}

\section{INTRODUCTION}

In the present paper we investigate tropical mixtures of two star tree metrics. Here, star tree metrics on $n$ taxa are star trees with $n$ leaves labeled 1 through $n$, equipped with nonnegative weights on all its edges. Tropical mixture of two metrics $D, \bar{D}$ on $n$ taxa are defined by point-wise maxima of metrics on $n$ taxa, i.e.

$$
(D \oplus \bar{D})_{(i, j)}=\max \left\{D_{(i, j)}, \bar{D}_{(i, j)}\right\} \text { for all } 1 \leq i, j \leq n .
$$

This study was inspired by the recent work of Matsen, Mossel and Steel on phylogenetic mixtures of trees ([8], [9]), as well as by some open questions regarding tropical secant varieties of linear spaces ([3], [4]).

Matsen and his collaborators consider phylogenetic mixtures (convex combinations of site pattern frequencies) of two weighted trees of the same topological type, and they characterize the conditions under which these give rise to weighted trees of a different topology. In particular, they pay special attention to mixtures of star tree metrics on four taxa, since tree topologies are characterized by the subtrees spanned by four of its leaves. Their interest in studying these mixtures lies in the branch repulsion phenomenon. More precisely, they observed that if we mix together two weighted trees on four taxa with the same topology, with short internal branch lengths and with pendant edges alternating being long and short, we are likely to get a tree with a different topology. Intuitively, this phenomenon implies that we can

2010 Mathematics Subject Classification. 52B70,(15A03,14M25).

Key words and phrases. Mixture models, (star) tree metrics, tropical secant varieties, toric varieties, phylogenetic trees.

The author was supported by a UC Berkeley Chancellor's Fellowship and the Laboratory for Mathematical and Computational Biology at UC Berkeley. 
approximate any tree topology we want by mixing together two star tree metrics. Thus, the interest in understanding mixtures of star tree metrics.

In this paper, rather than being interested in phylogenetic mixtures, we focus our attention on tropical mixtures of star tree metrics. Tropical mixtures arise naturally in the context of probability theory as approximations of mixtures of multivariate Poisson distributions ([11, Chapter 3]) and logdet transforms defined for Markov models on trees ([13, Theorem 8.4.3], [10]). Since log-limits of secant varieties of toric varieties correspond to tropical secant varieties of linear spaces, one should expect similar results between convex mixtures and tropical mixtures of star tree metrics. Theorem 2.8 shows this connection.

In the language of algebraic geometry, the image of the convex mixture map of two star tree metrics studied by Matsen et al. corresponds to secant varieties of star tree metrics. If we allow negative weights for the edges of our star trees, then mixtures of these objects correspond to secant varieties of star trees. Since the convex mixture map is polynomial, we can tropicalize both the space of star trees and the map to obtain a piecewise linear map: the tropical mixture map. Its image will be the tropical first secant of an $n$ dimensional linear subspace of $\mathbb{R}^{\left(\begin{array}{c}n \\ 2\end{array}\right)}$ corresponding to the tropicalization of the space of star trees ([3]). If we replace the space of star trees by the space of star tree metrics and we tropicalize both the space and the mixture map, we obtain a piecewise linear map parameterizing the first tropical secant of a polyhedral cone rather than of a linear space. The extremal rays of this cone are the cut metrics $d_{i}$ assigning $d_{i}(i, j)=1$ for all $j \neq i$ and where all other distances are zero. These cut metrics also span the space of star trees. The sign constraints on the coefficients of the linear combinations in this cone reflect the sign constraints on the edge weights of our star trees. While star trees admit edges with negative weights, this is forbidden in the biological framework of star tree metrics.

One interesting question that arises from these two settings involves star tree and star tree metric ranks of a symmetric matrix. Here, by star tree (resp. star tree metric) rank we mean the minimum number $k$ of star trees (resp. star tree metrics) required to express a given metric as a tropical mixture of $k$ star trees (resp. star tree metrics). By relaxing the positivity condition on the admissible edge weights, one can show that every tree metric on $n$ taxa can be written as the tropical mixture of $n-2$ star trees ([2, Theorem 5]). However, the analogous question for metrics and star tree metrics is more delicate. Indeed, we prove that most cut metrics on $n$ taxa (defined by partitions of the $n$ taxa, assigning pairwise distance 0 to pairs of taxa on the same subset, and distance 1 otherwise) are not tropical mixtures of finitely many star tree metrics. This implies that the set of star tree metric ranks is infinite.

The paper is organized as follows. In Section 2 we recall the basic definitions on distances over finite sets and tree metrics, and we present our main 
result: a complete characterization of tree metrics with star tree metric rank at most two, together with a description of the fibers of the mixture map (Theorem 2.8). All proofs are deferred to Section 3.

In Section 4 we switch gears and we describe the general framework of tropical secants of linear spaces as treated in $[3,4]$. In the case of star trees with arbitrary weights, the associated linear space is spanned by $n$ vectors $\left\{r_{i}=\sum_{j<i} e_{j i}+\sum_{j>i} e_{i j}, 1 \leq i \leq n\right\}$. In the spirit of [3, 4], we can asso-

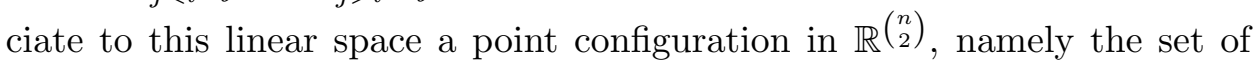
vertices of the well-studied second hypersimplex $\Delta(2, n)$. Tropical mixtures correspond to certain regular subdivisions of this polytope. Tropical secants of star tree metrics have the same underlying point configuration, but the crucial difference between these two settings lies in the admissible regular subdivisions of $\Delta(2, n)$, which we call positive regular subdivisions. Moreover, we show they are different even in the simplest case of trees on four taxa. In particular, the existence of metrics with infinite star tree metric rank implies that regular subdivisions of $\Delta(2, n)$ need not be positive. As a corollary, we show that the well-known problem of characterizing metrics on $n$ taxa with finite tree metric ranks cannot be approached by studying star tree ranks (see [11, Chapter 3] for a conjecture on this topic). We finish this paper with several open questions on tropical secant varieties of polyhedral cones, which provide the natural setting to investigate mixtures of tree metrics.

\section{BASIC DEFINITIONS AND MAIN RESUlT}

We begin by providing notation and basic definitions. We fix $[n]=$ $\{1, \ldots, n\}$.

Definition 2.1. A tree $T$ on $n$ taxa is a connected graph with no cycles and with leaves labeled by $[n]$. We let $E(T)$ be the edges of $T$.

It is well-known that trees are completely determined by their list of quartets, i.e. subtrees spanned by four leaves. For example, the quartet $(i j \mid k l)$ represents the subtree in Figure 1. Many authors have discussed

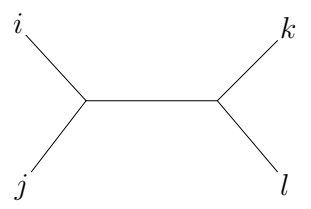

Figure 1. Subtree determined by the quartet $(i j \mid k l)$.

this tree representation and the minimum number of quartets required to characterize the topological type of a tree ([1], [6], [7, §5.4.2]).

Given a tree $T$, we can assign nonnegative weights to all edges in $T$ via a map $w: E(T) \rightarrow \mathbb{R}_{+}$. The pair $(T, w)$ is called a weighted tree. Throughout this section all trees are weighted unrooted trees on $n$ taxa. 
Given $i, j \in[n]$ and $(T, w)$ a tree, we define the distance between $i$ and $j$ to be $d_{T}(i, j)=\sum_{e \in(i \rightarrow j)} w(e)$, where $e$ varies along all edges in the unique path from $i$ to $j$. We omit the subscript $T$ when understood.

Definition 2.2. A dissimilarity map is a symmetric matrix $D \in \mathbb{R}_{+}^{n \times n}$ such that $D_{i i}=0$ for all $i \in[n]$. Alternatively, whenever it is convenient for us we encode $D$ by a vector in $\mathbb{R}_{+}^{\left(\begin{array}{c}n \\ 2\end{array}\right)}$ corresponding the upper triangular portion of the original symmetric matrix. A dissimilarity map defines a metric if it satisfies the triangular inequality $D_{i j} \leq D_{i k}+D_{k j}$ for all triples $i, j, k$.

Definition 2.3. A dissimilarity map $D$ is a tree metric if there exists a (unique) weighted tree $(T, w)$ such that $D=d_{T}$. By abuse of notation we sometimes denote a tree metric by its unique associated weighted tree.

From the definition, it is straightforward to check that the set of dissimilarity maps is a closed pointed rational polyhedral cone isomorphic to $\mathbb{R}_{+}^{\left(\begin{array}{c}n \\ 2\end{array}\right)}$. Likewise, the set of metrics is a full dimensional closed pointed rational polyhedral cone contained in the cone of dissimilarity maps. However, the space of tree metrics is a non-convex cone. It is a polyhedral complex, whose cells are convex cones. Each maximal cell is a closed rational polyhedral cone corresponding to a fixed tree topology and its degenerations (obtained by setting some edge lengths to be zero). Its extremal rays are the compatible split metrics $d_{A}$ defined by subsets $A$ of $[n]$, that determine the topology of the tree $T$. We define such split metrics as $d_{A}(i, j)=1$ if $\{i, j\} \not \subset A$ and $\{i, j\} \not \subset[n] \backslash A$, and 0 in all other cases. The compatibility condition among $d_{A}$ and $d_{A^{\prime}}$ says that one of the four intersections of $A$ or its complement and $A^{\prime}$ or its complement is empty. For a given tree topology, the cone spanned by the corresponding compatible split metrics is isomorphic to the orthant $\mathbb{R}_{+}^{|E(T)|-n}$ ([11, Prop 2.37]). Tree metrics are characterized algebraically by the "Four point condition" [11, Theorem 2.36]:

Theorem 2.4. (Four point condition) A dissimilarity map $D \subset R_{+}^{\left(\begin{array}{c}n \\ 2\end{array}\right)}$ is a tree metric if and only if for any 4 -tuple $i, j, k, l \in[n]$, the maximum among

$$
\left\{D_{i j}+D_{k l}, D_{i k}+D_{j l}, D_{i l}+D_{j k}\right\}
$$

is attained at least twice. In particular, a tree metric $D$ is realized by a star tree if and only if $D_{i j}+D_{k l}=D_{i k}+D_{j l}=D_{i l}+D_{j k}$ for any $i, j, k, l$.

In Section 4 we will view this cone as the nonnegative points in the tropicalization of a toric variety. Next, we define mixtures of dissimilarity maps:

Definition 2.5. Let $D, \bar{D}$ be two dissimilarity maps. We define the tropical mixture of $D$ and $\bar{D}$ as $(D \oplus \bar{D})_{i j}=\max \left\{D_{i j}, \bar{D}_{i j}\right\}=D_{i j} \oplus \bar{D}_{i j}$ for all $i, j \in[n]$. This generalizes to mixtures of any number of dissimilarity maps in the natural way.

To simplify notation, throughout the paper we refer to this construction as the mixture of $D$ and $\bar{D}$. From the definition it is straightforward to 
see that mixtures of metrics are metrics themselves. Since the Four point condition with non distinct indices provides the metric condition, we only need to check tuples of four distinct indices.

We define the mixture map as the function $\phi:\{\text { tree metrics }\}^{2} \rightarrow\{$ metrics $\}$ sending $(D, \bar{D}) \mapsto D \oplus \bar{D}$. Since $\lambda(D \oplus \bar{D})=(\lambda D) \oplus(\lambda \bar{D})$ for $\lambda \in \mathbb{R}_{+}$, it follows that the image of $\phi$ is also a pointed cone inside $\mathbb{R}_{+}^{\left(\begin{array}{c}n \\ 2\end{array}\right)}$. The commutativity of the mixture map can be interpreted by a natural action of the group $\mathbb{Z}_{2}$ on pairs of tree metrics, sending a pair $(D, \bar{D})$ to $(\bar{D}, D)$. The fibers of the mixture map will be a polyhedral complex that is closed under this action, mapping cells to cells. The fiber of $\phi$ over a generic point would be pure, of dimension two with at least two distinct orbits. We will see several examples of this behavior later on.

We now focus our attention on the restriction of $\phi$ to star tree metrics:

$$
\phi:\{\text { star tree metrics }\} \times\{\text { star tree metrics }\} \rightarrow\{\text { metrics }\} .
$$

The image of this restriction is also a pointed cone. The main goal of this paper is to characterize the set $\operatorname{im} \phi \cap\{$ tree metrics\}. Along the way, we determine the fibers of $\phi$. Since $D=D \oplus D$, we already know that $\{$ star tree metrics $\} \subset \operatorname{im} \phi$. Thus, we need only study when a non-star tree metric belongs to the image of $\phi$. We now present the desired characterization. Our building blocks with be the trees on four and five taxa.

Lemma 2.6. Assume $n=4$ and let $T$ be the quartet $(i j \mid k l)$ with edge weights $e_{i}, e_{j}, e_{k}, e_{l}$ and $g$ as in Figure 2a. Then, $T$ is a mixture of two star tree metrics if and only if $e_{i}, e_{j} \geq g$ or $e_{k}, e_{l} \geq g$. Moreover for each pair of inequalities, the fiber over $T$ is a polyhedral complex in $\mathbb{R}^{8}$ whose maximal cells are polyhedra of dimension at most two. Each maximal cell is affinely isomorphic to either a product of (degenerate) intervals, a (degenerate) trapezoid or a (degenerate) triangle in $\mathbb{R}^{2}$, and there are either eight or sixteen of them. The fibers are closed under the action of $\mathbb{Z}_{2}$ on pairs of star tree metrics.

Notice that the fibers over boundary points where some equality holds consist of degenerate star trees, because some edges have weight zero.

It is interesting to compare the result of the previous lemma with the analogous one for convex mixtures of two star trees on four taxa [8, Prop 2.2]. The tree metrics that are convex mixture of two star tree metrics are exactly the star trees and the quartet trees where the internal branch length is shorter than the sum of the weights of any pair of non-adjacent edges.

Lemma 2.7. Assume $n=5$ and let $T$ be a non-star weighted tree on five taxa. Denote by $e_{t}$ the weight of the edge attached to the leaf $t \in[n]$. Then, $T$ is a mixture of two star tree metrics if and only if $T$ has only one internal edge (labeled $g$ ), and $e_{i}, e_{j} \geq g$, where $i, j$ are the leaves of the unique cherry attached to one endpoint of the internal edge (see Figure 2b). The fiber of $\phi$ over such a tree $T$ is a polyhedral complex of dimension at most two with eight maximal cells, that is closed under the natural symmetry action of $\mathbb{Z}_{2}$. 
In particular, from the lemma we know that no trivalent tree metric is a mixture of two star tree metrics. The characterization for arbitrary number of taxa follows from the previous two lemmas.

Theorem 2.8. Let $T$ be a non-star tree metric. Then $T$ is a mixture of two star tree metrics if and only if one of the following conditions hold:

(i) $n=4, T$ is the quartet $(i j \mid k l)$ and $e_{i}, e_{j} \geq g$ or $e_{k}, e_{l} \geq g$, where $g$ denotes the weight of the internal edge (see Figure 2a);

(ii) $n \geq 5$, $T$ has only one internal edge with weight $g$, and if $I$ and $J$ are a partition of $[n]$ into labels of leaves attached to the two extremal points of the internal edge of $T$, and we assume $|I| \leq|J|$, we have two cases:

(a) $|I|=2$ and $e_{i_{1}}, e_{i_{2}} \geq g$ (see Figure 2b);

(b) $|I| \geq 3$, and $e_{i} \geq g$ for all $i \in[n]$ (see Figure 2c).

The fibers of $\phi$ over these trees are polyhedral complexes with eight or sixteen maximal cells of dimension at most two. These complexes are closed under the action of $\mathbb{Z}_{2}$. Over generic points, these fibers are pure complexes of dimension exactly two.

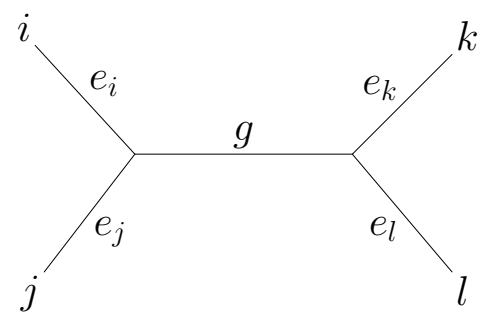

(a) $|I|=p=2=q=|J|$

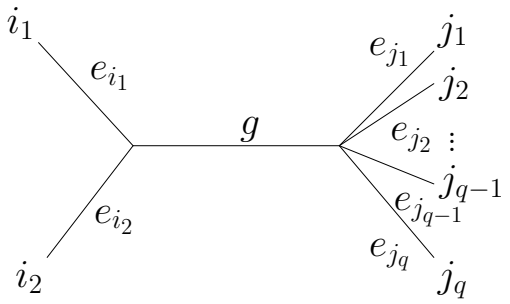

(b) $|I|=p=2<q=|J|$

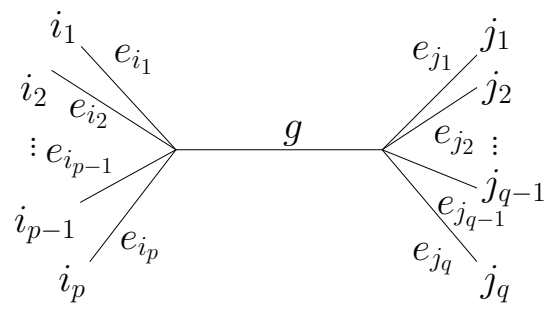

(c) $3 \leq|I|=p \leq q=|J|$

Figure 2. Tree topologies of mixtures of two star tree metrics.

\section{Proof of Lemmas 2.6, 2.7, And of Theorem 2.8}

Before presenting the proofs of the two main lemmas, we need an algebraic characterization of the topology of three metrics that equal $D \oplus \bar{D}$ in terms of the entries of the star tree metrics $D$ and $\bar{D}$. Since the topology of star 
trees is highly symmetric we need only treat the case of fibers of the mixture map over the quartet $(12 \mid 34)$. The general case follows by relabeling.

Following the proof technique in [9], our description involves relative differences among the entries of both metrics. We denote by $a, d, c$ and $e$ the weights of the edges in $D$ adjacent to leaves 1 through 4 . With the same convention, $\bar{a}, \bar{d}, \bar{c}$ and $\bar{e}$ are used for the edges in $\bar{D}$. We label the middle edge of the quartet (12|34) by $g$ (see Figure 2a). Note that since the image of $\phi$ and the trees with a fixed topology are cones, then our characterization must be invariant under multiplication by positive scalars.

From the edge weights in the star tree metrics $D$ and $\bar{D}$ we obtain two $4 \times 4$ nonnegative symmetric matrices. To simplify notation, we only show the coefficients of these matrices above the diagonal:

$$
D:=\left(\begin{array}{cccc}
0 & a+d & a+c & a+e \\
& 0 & d+c & d+e \\
& & 0 & c+e \\
& & & 0
\end{array}\right) ; \bar{D}:=\left(\begin{array}{cccc}
0 & \bar{a}+\bar{d} & \bar{a}+\bar{c} & \bar{a}+\bar{e} \\
& 0 & \bar{d}+\bar{c} & \bar{d}+\bar{e} \\
& & 0 & \bar{c}+\bar{e} \\
& & & 0
\end{array}\right) .
$$

We consider six new indeterminates $s, t, x, y, u$ and $w$, which express the relative differences between the matrices $D$ and $\bar{D}$.

$$
\text { (1) }\left\{\begin{array}{l}
\bar{a}+\bar{c}=a+c+s, \\
\bar{d}+\bar{e}=d+e+t,
\end{array},\left\{\begin{array}{l}
\bar{a}+\bar{e}=a+e+x, \\
\bar{d}+\bar{c}=d+c+y,
\end{array},\left\{\begin{array}{c}
\bar{a}+\bar{d}=a+d+u, \\
\bar{c}+\bar{e}=c+e+w .
\end{array}\right.\right.\right.
$$

From (1) we see that these new variables satisfy two linear equations:

$$
s+t=x+y=u+w .
$$

Denote $s^{+}=\max \{s, 0\}$ and similarly for the other five new variables. Observe that $T_{13}=\max \left\{D_{13}, \bar{D}_{13}\right\}=D_{13}+s^{+}$, and similarly for the other variables. The next result will be crucial in the proof of Lemma 2.6.

Proposition 3.1. With the above notation, $T$ is the quartet (12|34) if and only if $u^{+}+w^{+}<s^{+}+t^{+}=x^{+}+y^{+}$.

Proof. Follow immediately from Theorem 2.4.

3.1. Proof of Lemma 2.6. Our strategy is elementary and it is based on a careful case by case analysis. We let $T$ be the quartet $(12 \mid 34)$. We assume $T=D \oplus \bar{D}$, and we find necessary and sufficient conditions on the weights of $T$ for this equality to hold. Our unique restrictions are given by requiring all values of weights to be nonnegative. We show that these conditions suffice to explicitly construct $D$ and $\bar{D}$ from $T$. Proposition 3.1 will be our main tool throughout the proof.

Since the mixture map is symmetric on $D$ and $\bar{D}$, without loss of generality we may assume $s \geq 0$. Thus, we need to analyze two cases according to the sign of $t$.

If $t \geq 0$, we need $s+t=x+y$ and $s+t=x^{+}+y^{+}$. Thus $x, y \geq 0$. Likewise $s+t=u+w$ but $u+w \leq u^{+}+w^{+}<s+t$, a contradiction. Hence 
$t<0$. By switching the roles of $s$ and $t$ we also conclude that $s$ and $t$ are both nonzero. Without loss of generality, we may assume $s>0$ and $t<0$.

By the symmetry on $(12 \mid 34)$, we observe that both pairs $(s, t),(x, y)$ have coordinates of opposite sign. In addition, since $x+y=s+t<s$ and $s=x^{+}+y^{+}$, then we must have either $x=s, y=t$ or $x=t, y=s$. By analyzing (1) we conclude that either:

$s=x>0, t=y<0 \Rightarrow \bar{a}=a+s-\frac{w}{2}, \bar{c}=c+\frac{w}{2}, \bar{d}=d+u+\frac{w}{2}-s, \bar{e}=e+\frac{w}{2}$, or

$s=y>0, t=x<0 \Rightarrow \bar{a}=a+\frac{u}{2}, \bar{c}=c+s-\frac{u}{2}, \bar{d}=d+\frac{u}{2}, \bar{e}=e+x-\frac{u}{2}$.

Note that these two cases are symmetric with respect to the order two permutation that is the product of the transpositions (12) and (34).

We now consider the sign patterns of $u$ and $w$. Assume $u, w$ have opposite sign. Then from $u+w=s+t$ we conclude $u^{+}+w^{+}<s$ if and only if $(0 \leq u<s$ and $w=s+t-u<0)$ or $(0 \leq w<s$ and $u=s+t-w<0)$. In case both $u$ and $w$ have the same sign, we have no restrictions other than $u+w=s+t$.

Using Theorem 2.4, we can compute the weights of the edges of $T$ in terms of the variables $a, c, d, e, s, t, x^{+}, y^{+}, u^{+}$and $w^{+}$, following the labeling of Figure 2a with $i=1, j=2, k=3, l=4$.

$$
\left\{\begin{array}{l}
g=\frac{s-\left(u^{+}+w^{+}\right)}{2}, e_{1}=a+\frac{x^{+}+u^{+}}{2}, e_{2}=d+\frac{y^{+}-s+u^{+}}{2}, \\
e_{3}=c+\frac{y^{+}+w^{+}}{2}, e_{4}=e+\frac{x^{+}-s+w^{+}}{2}
\end{array}\right.
$$

Next, we compute each indeterminate in (2) according to our two sign patters for $x$ and $y$. Case 1 corresponds to $s=x>0$ and $t=y<0$, whereas Case 2 gives $s=y>0$ and $t=x<0$. We subdivide these two cases according to further sign constrains: $s>u \geq 0$ and $w \leq 0$ (Cases 1.1 and 2.1), $s>w \geq 0$ and $u \leq 0$ (Cases 1.2 and 2.2) or $u, w$ with the same sign (nonnegative in Cases 1.3 and 2.3 and nonpositive in Cases 1.4 and 2.4). In this way, we get expressions for all our indeterminates and we obtain necessary properties they must have.

Remark 3.2. Notice that Cases 1 and 2 are symmetric. More precisely, they are equivalent if we change the labels for leaves 1 and 2 by 3 and 4 respectively and we also change the variables $(a, d, u, w, x, y)$ by $(c, e, w, u, y, x)$, while leaving $s$ and $t$ fixed. One needs to make these replacements between suitable pairs: 1.1 \& 2.2, 1.2 \& 2.1, $1.3 \& 2.3$ and $1.4 \& 2.4$ respectively. Therefore, it suffices to study Cases 1.1 through 1.4. This correspondence will be crucial for the proof of Lemma 2.7.

- Case 1.1: Suppose $s>u \geq 0$ and $w \leq 0$, then

$$
g=\frac{s-u}{2}, \quad e_{1}=a+\frac{s+u}{2}, \quad e_{2}=d-\frac{s-u}{2}, \quad e_{3}=c, \quad e_{4}=e .
$$


Since $a \geq 0, u \geq 0$ the previous equations yield $e_{1} \geq g$. Similarly, by requiring all variables $\bar{a}, \bar{d}, \bar{e}, \bar{c}$ and $e_{k}$ to be nonnegative we conclude that $e_{1}, e_{2} \geq g, d \geq s-u-w / 2$ and $c, e \geq-w / 2$.

- Case 1.2: Suppose $s>w \geq 0$ and $u \leq 0$, then

$$
\begin{gathered}
g=\frac{s-w}{2}, \quad e_{1}=a+\frac{s}{2}, \quad e_{2}=d-\frac{s}{2}, \quad e_{3}=c+\frac{w}{2}, \quad e_{4}=e+\frac{w}{2} . \\
\text { In this case, from } a \geq 0 \text { and } w \geq 0 \text { we also have } e_{1} \geq g . \text { Analo- } \\
\quad \text { gously, from } u \leq 0 \text { and } 0 \leq w<s \text { we obtain additional necessary } \\
\quad \text { conditions: } d \geq s-u-w / 2 \text { and } e_{2}=d-s / 2 \geq g-u \geq g . \\
\text { - Case 1.3: Suppose } u, w \geq 0, \text { then } \\
g=\frac{s-(u+w)}{2}, e_{1}=a+\frac{s+u}{2}, e_{2}=d-\frac{s-u}{2}, e_{3}=c+\frac{w}{2}, e_{4}=e+\frac{w}{2} .
\end{gathered}
$$

As before, we have $e_{1} \geq g$, and we obtain the extra conditions $d \geq s-u-w / 2, e_{2} \geq s-u-w / 2-(s-u) / 2=g$.

- Case 1.4: Suppose $u, w \leq 0$, then

$$
g=\frac{s}{2}, \quad e_{1}=a+\frac{s}{2}, \quad e_{2}=d-\frac{s}{2}, \quad e_{3}=c, \quad e_{4}=e .
$$

Here we have $e_{1} \geq g, c, e \geq-w / 2$ and $d \geq s-u-w / 2$, thus $e_{2}=d-s / 2 \geq s / 2-u-w / 2 \geq g$.

From the previous analysis, we conclude that $s=x, t=y$ implies $e_{1}, e_{2} \geq$ $g$ and $d \geq s-u-w / 2$. Changing variables as stated in Remark 3.2, we get analogous results for Cases 2.1 through 2.4. In particular, we obtain $e_{3}, e_{4} \geq g$ and $e \geq s-w-u / 2$. Hence, if (12|34) is a mixture of two star tree metrics we conclude $e_{1}, e_{2} \geq g$ or $e_{3}, e_{4} \geq g$.

For the converse, we assume that $T$ is the quartet $(12 \mid 34)$ and that it satisfies $e_{1}, e_{2} \geq g$ or $e_{3}, e_{4} \geq g$. Our goal is to construct all possible pairs $D, \bar{D}$ giving $T=D \oplus \bar{D}$. From the "if direction" we know that such pairs $(D, \bar{D})$ belong to a polyhedral complex of dimension at most 2 . Each cell is parameterized by the variables $u, w$.

Assume that the condition $e_{3}, e_{4} \geq g$ does not hold. The, we claim that Cases 1.1 through 1.4 are admissible if and only if $e_{1}, e_{2} \geq g$. We already know the "only if direction" from the previous discussion. We now prove the converse:

- Case 1.1: Assume $e_{1}, e_{2} \geq g$. We want to determine the values of $a, c, d, e$ and $\bar{a}, \bar{c}, \bar{d}, \bar{e}$ so that $T=D \oplus \bar{D}$. From the previous analysis we easily obtain $c=e_{3}, d=e_{2}+g$ and $e=e_{4}$. On the other hand, we have $s=u+2 g, a=e_{1}-(s+u) / 2=e_{1}-g-u$, $\bar{a}=e_{1}+g-w / 2, \bar{c}=e_{3}+w / 2, \bar{d}=e_{2}-g+w / 2$ and $\bar{e}=e_{4}+w / 2$. The only conditions on $u, w$ are determined by the nonnegativity of all weights in $D$ and $\bar{D}$, namely, $u, w$ are free parameters satisfying $0 \geq w \geq-2 \min \left\{e_{2}-g, e_{3}, e_{4}\right\}$ and $e_{1}-g \geq u \geq 0$. The sign patterns for $s, t$ hold automatically by construction. Hence, our necessary and sufficient conditions are $e_{1}, e_{2} \leq g$. 
- Case 1.2: As in the previous case, if we assume $e_{1}, e_{2} \leq g$ we get $s=2 g+w, a=e_{1}-g-w / 2, c=e_{3}-w / 2, d=e_{2}+g+w / 2$, $e=e_{4}-w / 2, \bar{a}=e_{1}+g \bar{c}=e_{3}, \bar{d}=e_{2}-g+u$ and $\bar{e}=e_{4}$, where $u$ and $w$ are two free parameters satisfying $0 \geq u \geq g-e_{2}$ and $2 \min \left\{e_{3}, e_{4}, e_{1}-g\right\} \geq w \geq 0$. Again, our necessary and sufficient conditions are $e_{1}, e_{2} \geq g$ as well.

- Case 1.3: Suppose $e_{1}, e_{2} \geq g$. From scratch we have $\bar{c}=e_{3}$ and $\bar{e}=e_{4}$. We let $u, w \geq 0$ be two free parameters, so $s=2 g+u+w$, $a=e_{1}-g-u-w / 2, c=e_{3}-w / 2, d=e_{2}+g+w / 2, e=e_{4}-w / 2$, $\bar{a}=e_{1}+g$ and $\bar{d}=e_{2}-g$. In particular, the only restrictions over $u, w$ are $0 \leq w \leq 2 \min \left\{e_{3}, e_{4}, e_{1}-g\right\}$ and $0 \leq u \leq e_{1}-g-w / 2$.

- Case 1.4: Assume $e_{1}, e_{2} \geq g$. Then $s=2 g, a=e_{1}-g, c=e_{3}$, $d=e_{2}+g$ and $e=e_{4}$. Our free parameters are $u, w \leq 0$. We get $\bar{a}=e_{1}+g-w / 2, \bar{c}=e_{3}+w / 2, \bar{d}=e_{2}-g+u+w / 2$ and $\bar{e}=e_{4}+w / 2$. Therefore, $u, w$ must satisfy $0 \geq w \geq-2 \min \left\{e_{3}, e_{4}, e_{2}-g\right\}$ and $0 \geq u \geq g-e_{2}-w / 2$.

By the symmetries mentioned in Remark 3.2, we have similar results for Cases 2.1 through 2.4. The necessary and sufficient conditions are $e_{3}, e_{4} \geq g$ in case $e_{1}, e_{2} \geq g$ does not hold. We also obtain $u, w$ as free parameters and their linear restrictions are translations of the restrictions from the corresponding previous four cases.

Therefore, we have three possible scenarios for the fibers to be nonempty:

(i) $e_{1}, e_{2} \geq g$ does not hold. Then $e_{3}, e_{4} \geq g$ and the fiber of the mixture map is the union of the four families described by Cases 2.1 through 2.4 , and their orbits under the action of $\mathbb{Z}_{2}$ (which interchanges $D$ and $\bar{D}$ ). The fiber over such a point is a polyhedral complex with eight maximal cells (Cases 2.1 through 2.4 and their $\mathbb{Z}_{2}$-orbits). Each cell is affinely isomorphic to a product of intervals, a trapezoid or a triangle in $\mathbb{R}^{2}$. If $e_{1}, e_{2}>g$, the fiber over this point is a pure complex of dimension two, i.e. the maximal cells have dimension two.

(ii) $e_{3}, e_{4} \geq g$ does not hold. Then $e_{1}, e_{2} \geq g$ and the fiber of the mixture map is a polyhedral complex with eight maximal cells (Cases 1.1 through 1.4 and their $\mathbb{Z}_{2}$-orbits) of dimension at most two.

(iii) $e_{1}, e_{2}, e_{3}, e_{4} \geq g$. Then, the fiber is a polyhedral complex with sixteen maximal cells defined by Cases $1.1-2.4$ and their $\mathbb{Z}_{2}$-orbits.

As a reference for the proof of Lemma 2.7, we include the description of all eight cases below. The free parameters are $u, w$. Notice that in all cases, one of the star trees has at least three of its edge weights independent of the values of $u$ and $w$.

- Case 1.1: $e_{1}-g \geq u \geq 0$ and $0 \geq w \geq-2 \min \left\{e_{2}-g, e_{3}, e_{4}\right\}$,

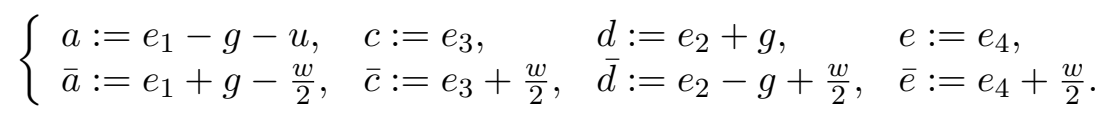


- Case 1.2: $0 \geq u \geq g-e_{2}$ and $2 \min \left\{e_{1}-g, e_{3}, e_{4}\right\} \geq w \geq 0$,

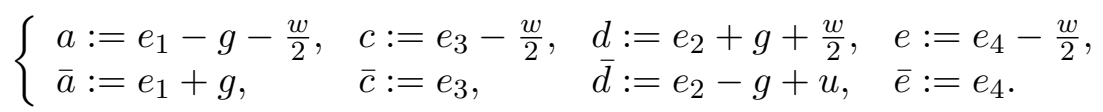

- Case 1.3: $e_{1}-g-w / 2 \geq u \geq 0$ and $2 \min \left\{e_{1}-g, e_{3}, e_{4}\right\} \geq w \geq 0$,

$\left\{\begin{array}{llll}a:=e_{1}-g-u-\frac{w}{2}, & c:=e_{3}-\frac{w}{2}, & d:=e_{2}+g+\frac{w}{2}, & e:=e_{4}-\frac{w}{2}, \\ \bar{a}:=e_{1}+g, & \bar{c}:=e_{3}, & \bar{d}:=e_{2}-g, & \bar{e}:=e_{4} .\end{array}\right.$

- Case 1.4: $0 \geq u \geq g-e_{2}-w / 2$ and $0 \geq w \geq-2 \min \left\{e_{2}-g, e_{3}, e_{4}\right\}$,

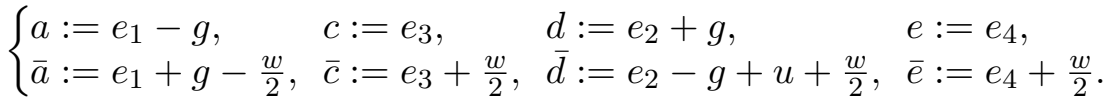

- Case 2.1: $2 \min \left\{e_{1}, e_{2}, e_{3}-g\right\} \geq u \geq 0$ and $0 \geq w \geq g-e_{4}$, $\left\{\begin{array}{llll}a:=e_{1}-\frac{u}{2}, & c:=e_{3}-g-\frac{u}{2}, & d:=e_{2}-\frac{u}{2}, & e:=e_{4}+g+\frac{u}{2}, \\ \bar{a}:=e_{1}, & \bar{c}:=e_{3}+g, & \bar{d}:=e_{2}, & \bar{e}:=e_{4}-g+w .\end{array}\right.$

- Case 2.2: $0 \geq u \geq-2 \min \left\{e_{1}, e_{2}, e_{4}-g\right\}$ and $e_{3}-g \geq w \geq 0$,

$\left\{\begin{array}{lll}a:=e_{1}, & c:=e_{3}-g-w, \quad d:=e_{2}, & e:=e_{4}+g, \\ \bar{a}:=e_{1}+\frac{u}{2}, & \bar{c}:=e_{3}+g-\frac{u}{2}, \quad \bar{d}:=e_{2}+\frac{u}{2}, & \bar{e}:=e_{4}-g+\frac{u}{2} .\end{array}\right.$

- Case 2.3: $2 \min \left\{e_{1}, e_{2}, e_{3}-g\right\} \geq u \geq 0$ and $e_{3}-g-u / 2 \geq w \geq 0$, $\left\{\begin{array}{llll}a:=e_{1}-\frac{u}{2}, & c:=e_{3}-g-\frac{u}{2}-w, & d:=e_{2}-\frac{u}{2}, & e:=e_{4}+g+\frac{u}{2}, \\ \bar{a}:=e_{1}, & \bar{c}:=e_{3}+g, & \bar{d}:=e_{2}, & \bar{e}:=e_{4}-g .\end{array}\right.$

- Case 2.4: $0 \geq u \geq-2 \min \left\{e_{1}, e_{2}, e_{4}-g\right\}$ and $0 \geq w \geq\left(g-e_{4}\right)-u / 2$, $\left\{\begin{array}{llll}a:=e_{1}, & c:=e_{3}-g, & d:=e_{2}, & e:=e_{4}+g, \\ \bar{a}:=e_{1}+\frac{u}{2}, & \bar{c}:=e_{3}+g-\frac{u}{2}, & \bar{d}:=e_{2}+\frac{u}{2}, & \bar{e}:=e_{4}-g+\frac{u}{2}+w .\end{array}\right.$

3.2. Proof of Lemma 2.7. As in the proof of Lemma 2.6, our goal is to study necessary and sufficient conditions under which a point has a nonempty fiber, and also to describe these fibers. By symmetry, we need only consider the two cases illustrated in Figure 3. We treat each topology separately, analyzing the possible quartets $(12 \mid 34),(12 \mid 35),(12 \mid 45)$ on these trees. Since we have a complete characterization of mixtures of two star tree metrics on four taxa, we construct our candidates for the mixtures on five taxa given each quartet on the trees. To finish, we glue our partial star tree metrics together (whenever possible) to build our candidates $D, \bar{D}$ on five taxa. We show this by an exhaustive case by case analysis for each quartet.

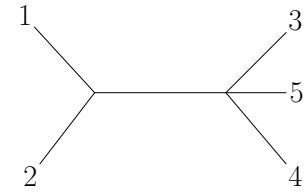

(a)

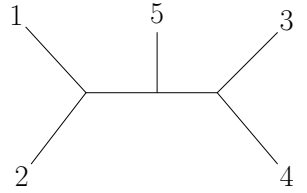

(b)

Figure 3. Non-star trees on five taxa, up to permutation of taxa. 
Proof for Figure 3a. Let $T$ be as in Figure 3a. As we said before, we have necessary and sufficient conditions for each quartet in $T$ to be a mixture of two star tree metrics. Assume $T=D \oplus \bar{D}$. Following the notation and proof-strategy of Lemma 2.6, we let $g$ be the weight of the unique internal edge in $T$ and $e_{i}$ the weight of the edge in $T$ adjacent to leaf $i$. Similarly, we let $a, d, c, e, f$ (resp. $\bar{a}, \bar{d}, \bar{c}, \bar{e}, \bar{f}$ ) be the weights of the edges in $D$ (resp. $\bar{D})$ adjacent to leaves 1 through 5 , as illustrated on the right-most picture in Figure 4. Since the quartets (12|34) and (12|35) involve leaves 1 and 3, we can always assume that $s>0$. However, for the quartet (12|45) we need to consider both signs for the difference $(\bar{a}+\bar{f})-(a+f)$. By considering the restrictions to the three mentioned quartets we see that the underlying tree $T$ must satisfy the following conditions:

$$
e_{1}, e_{2} \geq g \quad \text { or } \quad e_{3}, e_{4}, e_{5} \geq g
$$

Assume $e_{1}, e_{2} \geq g$. Therefore, we know that at least we have to analyze Cases 1.1 up to 1.4 for the three quartets, up to permuting $D$ and $\bar{D}$. We also treat Cases 2.1 through 2.4 if $e_{3}, e_{4} \geq g$.

Suppose (12|34) follows Case 1.1. Then, our free parameters $u, w$ satisfy $e_{1}-g \geq u \geq 0$ and $0 \geq w \geq-2 \min \left\{e_{2}-g, e_{3}, e_{5}\right\}$. The quartet (12|35) has the same value of $s=\bar{a}+\bar{c}-a-c>0$, and $u$, so we do not need to switch the order of $D$ and $\bar{D}$. We claim that the fiber over (12|35) can only correspond to Case 1.1. Assuming Cases 2.1 or 2.3, using that $g>0$ we see that $\bar{a}=e_{1}+g-w / 2>e_{1}=\bar{a}$, a contradiction. Likewise, for Cases 2.2 and 2.4 we obtain $\bar{a}=e_{1}+g-w / 2>e_{1}+u / 2=\bar{a}$, also a contradiction.

Next, we claim that any point in the fiber over the quartet (12|35) that satisfies Case 1.2, 1.3 or 1.4, must also satisfy Case 1.1. Call $v=\bar{c}+\bar{f}-c-f$. For Case 1.2 we obtain $u=w=v=0$ so the point in the fiber satisfies Case 1.1. Similarly, for Case 1.3 we get $w=v=0$, and the point also satisfies Case 1.1. Finally, for Case 1.4, we obtain $w=v$ and $u=0$, and the same conclusion holds. Thus by joining the weight values obtained for both quartets using Case 1.1 we get $v=w$ and compatible values for all weights in $D$ and $\bar{D}$.

To analyze the quartet $(12 \mid 45)$ we need to compute the difference $(\bar{a}+$ $\bar{f})-(a+f)$, since its sign determines the order of $D$ and $\bar{D}$ in expression (1). We obtain $(\bar{a}+\bar{f})-(a+f)=\left(e_{1}+e_{5}+g\right)-\left(e_{1}+e_{5}-g-u\right)=2 g+u>0$. Therefore, we do not need to switch the order of $D$ and $\bar{D}$ in our calculation. As before, there is only one possibility for the quartet (12|45): Case 1.1 must hold. The proof is analogous to the one of the quartet (12|34) because we know that the order of $D$ and $\bar{D}$ is preserved.

Therefore, if $e_{1}, e_{2} \geq g$ and we are in Case 1.1 for the quartet (12|34) we have that $T=D \oplus \bar{D}$ where

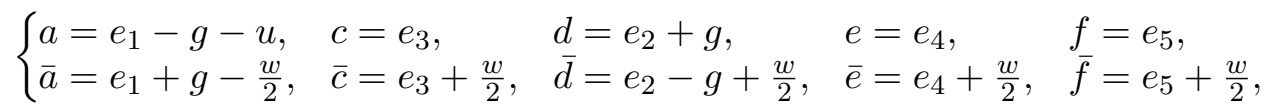


where $u, w$ are free parameters such that $e_{1}-g \geq u \geq 0,0 \geq w \geq$ $-2 \min \left\{e_{2}-g, e_{3}, e_{4}, e_{5}\right\}$.

On the other hand, assume Case 1.2 holds for (12|34). Keeping the notation of Case 1.1, a similar argument shows that $v=w, 0 \geq u \geq g-e_{2}$ and $2 \min \left\{e_{3}, e_{4}, e_{5}, e_{1}-g\right\} \geq w \geq 0$ on the quartets (12|34) and (12|35), with compatible values for all edge weights.

As before, to analyze the quartet $(12 \mid 45)$ we first need to compare $\bar{a}+\bar{f}$ with $a+f$. We have $\bar{a}+\bar{f}-(a+f)=2 g+w>0$, and so we do not need to permute $D$ and $\bar{D}$. Therefore, the only possibility for the quartet (12|45) is Case 1.2 which is compatible with the weights obtained before. Hence, $T=D \oplus \bar{D}$, where

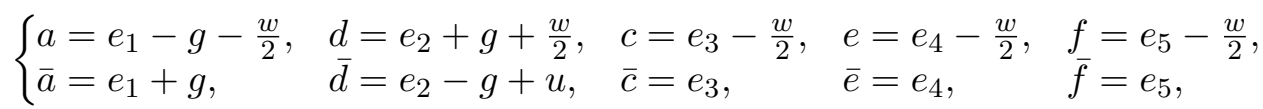

where $u, w$ are free parameters satisfying $0 \geq u \geq g-e_{2}$ and $2 \min \left\{e_{1}-\right.$ $\left.g, e_{3}, e_{4}, e_{5}\right\} \geq w \geq 0$.

Next, assume Case 1.3 holds for (12|34). As before, the same case must also hold for $(12 \mid 35)$ and we get $w=v$, where $2 \min \left\{e_{1}-g, e_{3}, e_{4}, e_{5}\right\} \geq w \geq 0$ and $e_{1}-g-w / 2 \geq u \geq 0$ and the values of all edge weights are compatible. In addition, $\bar{a}+\bar{f}-a-f=2 g+(u+w)=s>0$, so $D$ and $\bar{D}$ do not switch in the expression for the quartet (12|45). Like in the previous situation, Case 1.3 also holds for $(12 \mid 45)$ and we conclude $T=D \oplus \bar{D}$ with

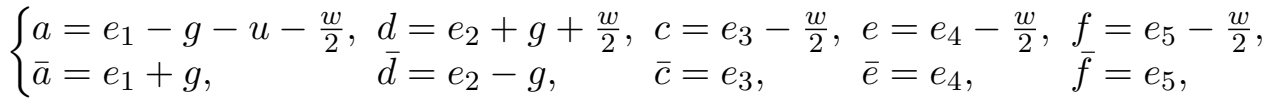

where $u, w$ are free parameters satisfying $2 \min \left\{e_{1}-g, e_{3}, e_{4}, e_{5}\right\} \geq w \geq 0$ and $e_{1}-g-w / 2 \geq u \geq 0$.

To finish, assume (12|34) satisfies Case 1.4. As before, we can easily see that the same case also holds for $(12 \mid 35)$ and we obtain compatible values for all weights. In this case, $\bar{a}+\bar{f}-(a+f)=2 g>0$, and (12|45) also satisfies Case 1.4. Therefore $T=D \oplus \bar{D}$ with

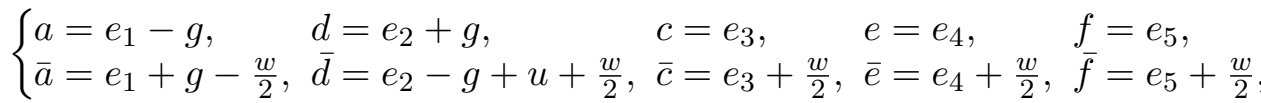
where $u, w$ are free parameters satisfying $0 \geq w \geq-2 \min \left\{e_{2}-g, e_{3}, e_{4}, e_{5}\right\}$ and $0 \geq u \geq g-e_{2}-w / 2$.

Next, we analyze Cases 2.1 through 2.4 for the quartet (12|34). We keep the notation from the previous discussion. By symmetry on the leaves 4 and 5, we know that Cases 2.1 through 2.4 also hold for the quartet (12|35).

Next, we argue that the quartet (12|34) cannot satisfy Cases 2.1 through 2.4. Assume the contrary. By looking at the weight $\bar{a}=e_{1}$ coming from the quartets $(12 \mid 34)$ and $(12 \mid 35)$, we see that all Cases 2.1 through 2.4 can hold for $(12 \mid 35)$, but they all imply that $\bar{a}+\bar{f}-(a+f) \leq-2 g<0$. Hence, for the expression of the quartet $(12 \mid 45)$ we have to switch the roles of $D$ and $\bar{D}$. We claim that Cases 1.1 through 1.4 are discarded for this 
quartet. By contraction, assume (12|34) satisfies Cases 2.1 or 2.3. Then by applying Cases 1.1 through 1.4 to the quartet $(12 \mid 45)$ we conclude that $\bar{d}>e_{2}=\bar{d}$, a contradiction. Similarly, if Cases 2.2 or 2.4 hold for (12|34) we get $d \geq e_{2}-g<e_{2}=d$, also a contradiction. Finally, by looking at the expression of $e$ coming from Cases 2.1 through 2.4 for the quartet (12|45), we obtain $e \leq e_{4}-g<e_{4}+g=e$, a contradiction. In conclusion, Cases 2.1 through 2.4 cannot hold for quartet (12|34).

From the previous analysis we conclude: if $e_{1}, e_{2} \geq g$, then the fiber over $T$ consists of Cases 1.1 through 1.4 for all three quartets. Moreover, they all satisfy the same case. Conversely, assume $T$ is the mixture of $D$ and $\bar{D}$. If $e_{1}, e_{2} \geq g$ does not hold, this would imply that Cases 2.1 through 2.4 are the only candidates for $(12 \mid 34)$. But from the construction this cannot happen. Therefore, we conclude that the fiber of the mixture map over a point coming from a tree metric on five taxa with a single internal edge is nonempty if and only if $e_{1}, e_{2} \geq g$.

Proof for Figure 3b. As in the previous discussion, we label the edges of $T$ and of the star trees as in Figure 4. In what follows, we show that no tree
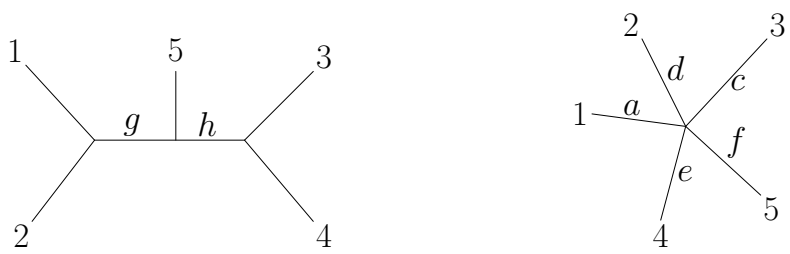

Figure 4 . Weights for edges in $T$ and in the star tree for $n=5$.

metric with this topology is a mixture of two star tree metrics.

Assume the contrary, and let $T$ be such a point. Then by considering the quartet $(12 \mid 34)$ we know that $e_{1}, e_{2} \geq g+h$ or $e_{3}, e_{4} \geq g+h$. Without loss of generality we may assume $e_{1}, e_{2} \geq g+h$. Now, consider the quartet (12|35). Since $e_{1}, e_{2} \geq g+h \geq g$, we have to analyze all eight cases for (12|34) and $(12 \mid 35)$.

Suppose Cases 1.1 or 1.4 hold for the quartet (12|34), so $d=e_{2}+g+h$. If Cases 1.1 or 1.4 hold for (12|35), we get $e_{2}+g=d=e_{2}+g+h$, because the middle edge weight for (12|35) is $g$. Similarly, for Cases 1.2 and 1.3 we get $\bar{a}=e_{1}+g+h-w / 2=e_{1}+g$ with $w \leq 0$ and $h>0$. Both situations lead to a contradiction. Likewise, for Cases 2.1 and 2.3, we get $d=e_{2}+g+h=e_{2}-u / 2$ where $u \geq 0$, which cannot occur, whereas Cases 2.2 and 2.4 yield $d=e_{2}+g+h=e_{2}$, also a contradiction. Thus, Cases 1.1 and 1.4 are not admissible for the quartet $(12 \mid 34)$.

Next, suppose Cases 1.2 or 1.3 are satisfied by (12|34). Then if we assume Cases 1.1 or 1.4 for the quartet (12|35) we get $d=e_{2}+g=e_{3}+g+h+\frac{w}{2}$

with $w \geq 0$ and $h>0$, a contradiction. Likewise, for Cases 1.2 and 1.3 we get $\bar{a}=e_{1}+g+h=e_{1}+g$ which cannot happen. For Cases 2.1 and 2.3 we 
get $\bar{a}=e_{1}+g+h=e_{1}$, and Cases 2.2 and 2.4 yield $\bar{a}=e_{1}+g+h=e_{1}+u / 2$ where $u \leq 0$, which cannot occur.

Finally, we analyze the Cases 2.1 through 2.4 for the quartet (12|34). By the previous discussion, we know that the quartet (12|35) can only satisfy Cases 2.1 through 2.4 as well. As in the previous discussion, we work with the weights $\bar{c}$ and $\bar{a}$ and we get contradictions for all possible expressions of the quartet (12|35). This concludes our proof.

Remark 3.3. Note that in the proof of Figure 3a, we found that all the quartets (12|34), (12|35) and (12|45) satisfy the same case (either 1.1, 1.2, 1.3 or 1.4) and the fiber is a polyhedral complex whose maximal cells have dimension at most two. The free parameters involved on different quartets are the same when restricting to each quartet, i.e. $w=v$ in the notation of the proof. This observation will be essential to prove Theorem 2.8.

3.3. Proof of Theorem 2.8. It remains to prove the item (ii) when $n \geq 6$. From Lemma 2.7, it is clear that we have only one topology on $n$ taxa that gives a tree metric in the image of the mixture map, besides the star tree topology. This topology is the one where the tree has a single internal edge, and it is illustrated in Figure 5. It is constructed by gluing two star trees with labels in the disjoint sets $I=\left\{i_{1}, \ldots, i_{p}\right\}$ and $J=\left\{j_{1}, \ldots, j_{q}\right\}$ with $I \sqcup J=[n]$. As before, we let $g$ be the weight of the unique internal edge and $e_{k}$ be the weight of the edge adjacent to the leaf $k \in[n]$. By symmetry

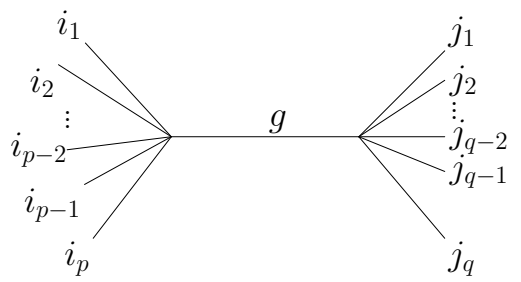

Figure 5. Non-star candidate $T$ for a mixture of two star tree metrics on $n$ taxa.

on the taxa, we can assume $1,2 \in I$ and $3,4 \in J$. Moreover, we assume $|I|=p \leq q=|J|$. We claim that we have only two cases to deal with, namely $|I|=2$ or $|I|>2$.

We first restrict the tree metric to the quartets $(12 \mid 34)$ and $(12 \mid 3 j)$, with $j>4$. Then, as we saw in the proof of Lemma 2.7 and in Remark 3.3, both quartets satisfy the same case (among Cases 1.1 through 1.4) and $e_{1}, e_{2} \geq g$. Likewise, the same case will hold for all quartets $(12 \mid k l)$ where $k, l \in J$.

Assume $|I|=2$. By gluing the previous partial solutions together we get that the fiber over a tree metric with a single internal edge is a polyhedral complex with eight maximal cells of dimension at most two if and only if $e_{1}, e_{2} \geq g$. All other non-star tree metrics have empty fibers.

Now, we assume $|I|>2$. By switching the roles of $I$ and $J$ we conclude that the fiber is nonempty if and only if $e_{i} \geq g$ for all $i \in[n]$ and that all 
the quartets $(k l \mid 34)$ (resp. $(12 \mid k l)$ ) with $k, l \in I$ (resp. $k, l \in J$ ) satisfy the same case among Cases 2.1 through 2.4 (resp. Cases 1.1 through 1.4). The correspondence between Cases 1 and 2 mentioned in Remark 3.2 guarantees that we can construct $D$ and $\bar{D}$ as in the case of five taxa. Thus, the fiber over such tree metric is a polyhedral complex with eight maximal cells of dimension at most two that is closed under the natural action of $\mathbb{Z}_{2}$. Each one of these maximal cells is isomorphic to a product of intervals, a trapezoid or a triangle in $\mathbb{R}^{2}$.

\section{Tropical SECANT VARIETIES AND the SPACE of StaR trees}

In this section, we identify mixtures of finitely many star tree metrics with relate tropical secant varieties of the cone of star tree metrics. For the basic definitions on tropical geometry we refer the reader to [12]. For a gentle introduction to applications of tropical geometry to phylogenetics, we recommend [11, Chapters 2-3].

In [14, Theorem 3.4], Speyer and Sturmfels showed that the space of (possibly negatively) weighted trees on $n$ taxa is (up to sign) the tropicalization of the classical Grassmannian $\mathscr{G}(2, n)$, called the tropical Grassmannian. In particular, the space of star trees is the tropicalization of the toric variety parameterized by the monomial map:

$$
\psi: \mathbb{C}\left[t_{1}^{ \pm 1}, \ldots, t_{n}^{ \pm 1}\right] \rightarrow \mathbb{C}\left[x_{i j}^{ \pm 1}: 1 \leq i<j \leq n\right] \quad \psi_{i j}(\underline{t})=\alpha_{i j} t_{i} t_{j},
$$

for fixed scalars $\alpha_{i j} \in\left(\mathbb{C}^{*}\right)\left(\begin{array}{l}n \\ 2\end{array}\right)$. This toric variety is cut out by the ideal

$$
\begin{aligned}
I & =\left\langle x_{i j}-\alpha_{i j} t_{i} t_{j}: 1 \leq i<j \leq n\right\rangle \bigcap \mathbb{C}\left[x_{i j}^{ \pm 1}: 1 \leq i<j \leq n\right] \\
& =\left\langle x_{i j} x_{k l}-\frac{\alpha_{i k} \alpha_{k l}}{\alpha_{i k} \alpha_{j l}} x_{i k} x_{j l} ; x_{i l} x_{j k}-\frac{\alpha_{i k} \alpha_{k l}}{\alpha_{i k} \alpha_{j l}} x_{i l} x_{j k}: i, j, k, l\right\rangle,
\end{aligned}
$$

where we identify $x_{i j}$ with $x_{j i}$ and $\alpha_{j i}$ with $\alpha_{i j}$ if $j<i$, and similarly for the other variables. The $n$-dimensional linear space $L$ spanned by the star trees is the tropical variety of tropical rank one symmetric matrices. The space of star tree metrics is the intersection of $L$ with the positive orthant.

From expression (3) we know that $L$ is generated by the set of all vertices of the second hypersimplex $\Delta(2, n) \subset \mathbb{R}^{n}$, i.e. the exponents $\left\{e_{i}+e_{j}: 1 \leq\right.$ $i<j \leq n\}$ of our monomial map $\psi$. The polytope has $\left(\begin{array}{l}n \\ 2\end{array}\right)$ vertices and dimension $n-1$. We refer to [15, Chapter 9], [5, Chapter $6 \S 3$ ] for the historical background and basic properties of this well-studied polytope.

As we mentioned in Section 1, the mixtures of $k$ star tree metrics are the points in the $k$-tropical secant of the space of star tree metrics. Due to the positivity restriction on the edge weights, tropical secants of cones define the appropriate setting to analyze mixtures of finitely many star tree metrics, as we now show. Given a cone $\mathscr{L}$ in $\mathbb{R}^{m}$, we define its $k$-tropical secant as the collection of points $x$ in $\mathbb{R}^{m}$ that can be written as

$$
x_{i}=\left(p^{(1)} \oplus \ldots \oplus p^{(k)}\right)_{i}:=\max \left\{p_{i}^{(1)}, \ldots, p_{i}^{(k)}\right\} \quad \forall i \in[n],
$$


for suitable points $p^{(1)}, \ldots, p^{(k)}$ in $\mathscr{L}$. Following the notation of $[3$, Theorem 2.1], we replace the linear space $L$ by a rational polyhedral cone. Likewise, the generators of $L$ are replaced by the extremal rays of this cone. Our approach follows the $\max$ convention for the tropical semiring, as opposed to the min convention of [3]. Among the many results of [3] that can be extended to the cone setting, we have:

Theorem 4.1. Let $\mathscr{L} \subset \mathbb{R}^{m}$ be a polyhedral cone with d extremal rays. Consider the associated matrix $M_{\mathscr{L}} \in \mathbb{R}^{d \times m}$ whose rows equal the previous $d$ rays. Let $V_{\mathscr{L}}=\left\{v_{1}, \ldots, v_{m}\right\}$ be the $m$-point configuration of $\mathbb{R}^{d}$ given by the columns of $M_{\mathscr{L}}$. Then a vector $x \in \mathbb{R}^{m}$ is in the $k$-th tropical secant of $\mathscr{L}$ (with the $\max$ convention) if and only if the lower envelope of the polytope formed by the height vector $x$ has $k+1$ facets given by linear functionals with only positive coefficients whose union contains each point of $V_{\mathscr{L}}$.

Notice that points in the various tropical secants of $\mathscr{L}$ define special regular subdivisions of the polytope generated by $V_{\mathscr{L}}$. The positivity condition for the coefficients comes from the fact that we work with cones rather than linear spaces. For simplicity, we call such regular subdivisions positive regular subdivisions. Other than these small differences, the proof follows line by line the proof of $[3$, Theorem 2.1]. The cone $\mathscr{L}$ of star tree metrics has $n$ extremal rays, namely, the cut metrics corresponding to the partition $\{i\} \bigsqcup([n] \backslash\{i\})$, as we vary $i \in[n]$. These metrics are defined as $D_{i}(i, j)=1$ for $j \neq i$ and 0 otherwise. The integer matrix $M_{\mathscr{L}}$ is the matrix of exponents of the monomial map $\psi$ from (3).

Another interesting question to study is the relationship between the mixtures of star tree metrics, the tropical secants of cones and the secondary fan of $\Delta(2, n)$. By [3, Theorem 2.1, Corollary 2.2] we know that each regular subdivision in the $k$-th tropical secant variety of the space of star trees corresponds to a polyhedral cone of height vectors. In particular, this yields a decomposition of this tropical secant variety as a polytopal complex. In the case of the $k$-th tropical secant variety of a pointed cone we have:

Corollary 4.2. The $k$-th tropical secant variety of a pointed cone $\mathscr{L}$ is a cone from $\mathscr{L}$ over a polytopal complex, which we call the $k$-th tropical secant complex of $\mathscr{L}$. The faces correspond to positive regular subdivisions of $V_{\mathscr{L}}$ in which there exist $k+1$ facets containing all of the points, where a face $F$ contains a face $G$ if the regular subdivision associated to $F$ refines the one of $G$.

Consider the spaces of star trees, of star tree metrics, and their corresponding tropical secant complexes. We know that the complex corresponding to star tree metrics is strictly contained in the complex corresponding to star trees. In particular, it would be desirable to study how the cell structures of both complexes relate. In addition, one can investigate how the cell structure of this complex restricted to the space of tree metrics compares to the subdivision of the tropical Grassmannian $\mathscr{G}(2, n)$ into topological types. 
In [2], Cartwright and Chan study the possible star tree ranks of dissimilarity maps (see Definition 2.2). These ranks are defined as the minimum $r$ such that any dissimilarity map can be written as a mixture of $r$ star trees. If no such $r$ exists, we say that the matrix has infinite star tree rank. We define the star tree metric rank of a dissimilarity map in an analogous way. It follows from the definition that the star tree metric ranks are bounded below by the star tree ranks.

In [2, Theorem 5] the authors show that for $n \geq 3$, any dissimilarity map on $n$ taxa can be written as the mixture of $n-2$ star trees, possibly with negative weights on their edges. Moreover, this bound is tight. By comparing their result with Lemma 2.6, we see that any metric on four taxa is a tropical mixture of two star trees, but not necessarily two star tree metrics.

We now rephrase these properties in the language of tropical geometry. Since the configuration of points associated to $\Delta(2, n)$ is in convex position, [3, Corollary 2.3] shows that for $k \gg 0$, the $k$-th tropical secant (called the $\infty$-th tropical secant) of the space of star trees equals the ambient space

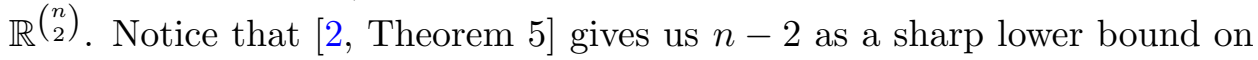
such $k$ 's. However, the space of tree metrics does not equal the $\infty$-th tropical secant variety of the star tree metrics. For example, Lemma 2.6 implies that the cut metric $D$ defined by the quartet $(12 \mid 34)$, where $D(1,3)=D(1,4)=$ $D(2,3)=D(2,4)=1$ and 0 otherwise, has star tree metric rank bigger than two. Furthermore, this rank is infinite. By contradiction, assume that for some $N \geq 1$ we can write $D=\bigoplus_{k=1}^{N} S_{k}$, where all $S_{k}$ are star tree metrics. For each $k, i$, we let $e_{i}^{(k)}$ be the weight of the edge pendant to leaf $i$ in $S_{k}$. Then we have $S_{k}(1,2)=e_{1}^{(k)}+e_{2}^{(k)} \leq D(1,2)=0$ and $S_{k}(3,4)=e_{3}^{(k)}+e_{4}^{(k)} \leq D(3,4)=0$. Since all weights are nonnegative, we conclude $e_{i}^{(k)}=0$ for all $i, k$, hence $D=0$, a contradiction. This example can be extended to higher number of taxa, by considering any cut metric given by a partition of $[n]$ where, at least, two subsets have more than one element. In particular, these results together with [3, Corollary 2.3] imply that regular subdivisions of the second hypersimplex need not be positive, as one would expect from the constructions.

As we see from the previous discussion, mixtures of star trees and of star tree metrics have completely different behavior, although the underlying combinatorics in both cases are closely related. Mixtures of star trees were extensively studied in [2], but basic questions on the mixtures of star tree metrics remain open. Among these are the characterization of $k$-mixtures of star tree metrics for $k \geq 3$, and the lack of effective membership tests for these sets. We believe that a serious study of tropical secants of cones will help us to attack most of these open problems. 


\section{ACKNOWLEDGMENTS}

This project developed from a course project in Lior Pachter's Math 239 graduate course at UC Berkeley. I wish to thank Lior Pachter and Bernd Sturmfels for their guidance and suggestions, and also Dustin Cartwright for useful discussions. Finally, I thank the anonymous referee for careful reading and many clarifying suggestions.

\section{REFERENCES}

[1] D. Bryant and M. Steel. Fast algorithms for constructing optimal trees from quartets. In SOSA'99: Proceedings of the tenth annual ACM-SIAM symposium on Discrete algorithms, pages 147-155, Philadelphia, PA, USA, 1999. Society for Industrial and Applied Mathematics.

[2] D. Cartwright and M. Chan. Tropical rank of symmetric matrices. In press, Combinatorica, 2011.

[3] M. Develin. Tropical secant varieties of linear spaces. Discrete and Computational Geometry, 35(1):117-129, 2006.

[4] J. Draisma. A tropical approach to secant dimensions. J. Pure Appl. Algebra, 212(2):349-363, 2008.

[5] I. Gel'fand, M. Kapranov, and A. Zelevinsky. Discriminants, Resultants, and Multidimensional Determinants. Birkhäuser, 1994.

[6] S. Grunewald, K. Huber, V. Moulton, and C. Semple. Encoding phylogenetic trees in terms of weighted quartets. Journal of Mathematical Biology, 56:465-477, 2008.

[7] T. Hodkinson, J. Parnell, and C. Wai-Kai, editors. Reconstructing the Tree of Life: Taxonomy and Systematics of Species Rich Taxa. Systematics Association Special Volumes. CRS Press, 2007.

[8] F. Matsen, E. Mossel, and M. Steel. Mixed-up trees: the structure of phylogenetic mixtures. Bull. Math. Biol., 70(4):115-1139, 2008.

[9] F. Matsen and M. Steel. Phylogenetic mixtures on a single tree can mimic a tree of another topology. Sys. Bio., 56(5):767-775, 2007.

[10] L. Pachter and D. Speyer. Reconstructing trees from subtree weights. Appl. Math. Lett., 17:512-621, 2004.

[11] L. Pachter and B. Sturmfels, editors. Algebraic statistics for computational biology. Cambridge University Press, 2005.

[12] J. Richter-Gebert, B. Sturmfels, and T. Theobald. First steps in tropical geometry. In G. Litvinov and V. Maslov, editors, Idempotent Mathematics and Mathematical Physics, volume 377 of Contemporary Mathematics, pages 289-317. AMS, 2005.

[13] C. Semple and M. Steel. Phylogenetics. Oxford University Press, 2003.

[14] D. Speyer and B. Sturmfels. The tropical Grassmannian. Adv. Geom., 4(3):389-411, 2004.

[15] B. Sturmfels. Gröbner bases and convex polytopes, volume 8 of University Lecture Series. AMS Bookstore, 1996.

E-mail address: macueto@math.columbia.edu

Mathematics Department, Columbia University, New York, NY, 10027, USA 